\title{
Influencia del estrés percibido en la función sexual de hombres y mujeres jóvenes
}

\author{
Rafael Bernal Mohedano \\ al340193@uji.es \\ Carlos García Montoliu \\ al340185@uji.es \\ Jaime Calderón Benítez \\ al340170@uji.es \\ Antonio José Castell Domínguez \\ al225794@uji.es \\ Estefanía Ruiz Palomino \\ eruiz@uji.es
}

\section{Resumen}

Introducción: La literatura científica demuestra el impacto negativo que el estrés tiene en la función sexual masculina y femenina. Es por ello que el objetivo del presente estudio es evaluar los efectos del estrés en la función sexual de hombres y mujeres jóvenes.

Método: Para ello se administró un ítem como autoinforme del nivel de estrés diario percibido, el Índice Internacional de Función Eréctil y el Índice de Función Sexual Femenina a 907 jóvenes con edades comprendidas entre los 18 y los 35 años ( $M=24,38 ; D T=3,97$ ).

Resultados: Los resultados muestran relaciones estadísticamente significativas entre el estrés y los componentes de excitación sexual $(p \leq 0,001)$, lubricación $(p=0,004)$, orgasmo $(p=0,003)$, satisfacción sexual $(p=0,005)$ y dolor $(p=0,029)$ en las mujeres; y de satisfacción general $(p=0,020)$ en los hombres.

Conclusiones: Sería importante incluir la evaluación del estrés en el asesoramiento y tratamiento de los problemas sexuales, especialmente en mujeres, así como dotar de estrategias de afrontamiento del estrés adaptativas a nivel preventivo.

Palabras clave: estrés, función sexual masculina, función sexual femenina, jóvenes, género. 


\section{Abstract}

Introduction: The scientific literature demonstrates the negative impact that stress has on male and female sexual function. The aim of this study is to assess the effects of stress on the sexual function of young males and females.

Method: A self-report item that assess the level of daily perceived stress, the International Index of Erectile Function and the Index of Female Sexual Function was administered to 907 young people aged between 18 and 35 years old $(M=24.38, S D=3.97)$.

Results: The results show statistically significant relationships between stress and the components of sexual arousal $(p \leq 0.001)$, lubrication $(p=0.004)$, orgasm $(p=0.003)$, sexual satisfaction $(p=0.005)$ and pain $(p=0.029)$ in females; and general satisfaction $(p=0.020)$ in males.

Conclusions: It would be important to include stress assessment in the counseling and treatment of sexual problems, specially in females, as well as to provide adaptive stress coping strategies at a preventive-focus interventions.

Keywords: stress, male sexual function, female sexual function, youth, gender.

\section{Introducción}

Selye (1936) definió el estrés como una «respuesta biológica no específica que generan todos los organismos frente a las demandas y presiones del medio ambiente». Actualmente, pese a que no hay una definición de estrés globalmente aceptada por toda la comunidad científica, gran parte de los autores explican este concepto en base a cuatro características principales: se produce una respuesta corporal, esta respuesta tiene lugar en base a demandas físicas o ambientales, las demandas pueden ser externas o internas y exceden la capacidad del individuo para hacerles frente (García-Herrero, Mariscal, Gutiérrez y Ritzel 2013, 114). Las consecuencias sobre la salud física y mental de tener elevados niveles de estrés dependen en parte de las características genéticas y psicológicas de cada individuo, las cuales pueden actuar como factor de vulnerabilidad o de resistencia (Duque Moreno 2017, 30). Por un lado, el estrés puede favorecer el desarrollo de problemas de salud mental tales como el trastorno de ansiedad generalizada, el trastorno depresivo mayor, el trastorno de estrés postraumático o los trastornos relacionados con el consumo de sustancias (Thoits 2010, 42). Respecto a la salud física, la exposición prolongada a niveles elevados de estrés puede dar lugar a patologías de carácter orgánico como, por ejemplo, enfermedades cardiovasculares (Gianaros y Wager 2015, 315). Además, este factor también está relacionado con el desarrollo de obesidad y puede favorecer la aparición de conductas nocivas para la salud tales como consumir comida con un índice elevado de grasa, fumar o consumir drogas y otras sustancias (Hostinar, Nusslock y Miller 2018).

En el ámbito de la sexualidad, el estrés puede repercutir de manera negativa en la salud sexual de las personas favoreciendo el desarrollo de disfunciones sexuales como, por ejemplo, dificultades con el orgasmo, problemas de lubricación, síntomas pélvicos, incontinencia urinaria durante el sexo, evitación sexual, dispareunia o dolor durante el coito y deseo sexual hipoactivo en mujeres, o eyaculación precoz, 
eyaculación retardada y disfunción eréctil en hombres, así como disminución del deseo sexual y problemas reproductivos en ambos sexos (Abedi, Afrazeh, Javadifar y Saki 2015; Bodenmann, Ledermann, Blattner y Galluzzo 2006; Bodenmann, Atkins, Schär y Poffet 2010; Galanakis et al. 2015; Kelley y Gidycz 2019; Kirby 2011; Shifren, Monz, Russo, Segreti y Johannes 2008). Asimismo, algunos estudios señalan una relación negativa entre el nivel de estrés y la satisfacción sexual tanto de las personas evaluadas como de sus parejas sexuales (Leavitt, McDaniel, Maas y Feinberg 2017). El cortisol puede jugar un papel fundamental en el desarrollo de problemas sexuales, ya que varios estudios señalan la existencia de una relación positiva entre el nivel de estrés, el nivel de cortisol y la actividad del sistema nervioso simpático (Hamilton y Meston 2013). No obstante, el estrés puede afectar en mayor o menor grado en base a ciertas variables personales de cada individuo. Bodenmann, Atkins, Schär y Poffet (2010) mostraron una mejor salud sexual en las personas que, pese a estar sometidas a niveles elevados de estrés, tenían buenas estrategias de afrontamiento.

En relación con el género, la literatura científica indica que las mujeres presentan niveles de estrés autopercibido más elevados que los hombres (Romero 2009; Ip, Lindfelt, Tran, Do y Barnett 2018). Algunos estudios señalan que hombres y mujeres utilizan estrategias de afrontamiento diferentes: mientras que las mujeres utilizan estilos de afrontamiento de tipo social, como la búsqueda de apoyo, los hombres, por su parte, usan estrategias de afrontamiento con un enfoque más cognitivo, como la reevaluación positiva o la planificación (González Cabanach, Fariña, Freire, González y Ferradás 2015, 21). A nivel neurológico, también se producen cambios diferenciales en hombres y mujeres ante la presencia de estrés. En hombres, la aparición de estrés se asocia con un incremento del flujo sanguíneo cerebral en el córtex prefrontal derecho y una reducción del mismo en el córtex orbitofrontal izquierdo, mientras que en las mujeres se activa principalmente el sistema límbico, incluyendo el estriado ventral, putamen, ínsula y córtex cingulado (Wang et al. 2007, 230).

Estas diferencias de género en cuanto al nivel de estrés autopercibido y a las estrategias de afrontamiento utilizadas, así como los cambios diferenciales que se producen a nivel neurológico, podrían suponer una afectación distinta en la función sexual masculina y femenina ante la presencia de estrés, ya que diversos estudios exponen una relación directa entre una mejor salud sexual y factores como, por ejemplo, el empleo de estrategias de afrontamiento adaptativas (Crisp et al. 2013, 73). Es por esto que el objetivo principal del presente estudio es analizar la relación entre el nivel de estrés autopercibido y los diferentes elementos que conforman la función sexual tanto en hombres como en mujeres jóvenes.

En base al objetivo propuesto, se plantean dos hipótesis:

- H1: Las mujeres presentarán un nivel mayor de estrés autopercibido que los sujetos de sexo masculino.

- H2: Ante la presencia de niveles elevados de estrés, existirán diferencias en la afectación de la función sexual en función del género.

\section{Método}

\section{Participantes}

La muestra de este estudio está constituida por 907 jóvenes con edades comprendidas entre los 18 y los 35 años $(M=24,38 ; D T=3,97)$. En cuanto al género, de la muestra total, 575 son hombres $(63,4 \%)$ y 332 son mujeres $(36,4 \%)$. La mayoría de los participantes eran españoles $(94,8 \%)$, tenían estudios superiores $(56,7 \%)$, 
estaban solteros $(91,8 \%)$, tenían pareja estable en el momento de la evaluación $(60,6 \%)$, eran ateos o agnósticos $(66,6 \%)$ y se definían como heterosexuales $(88,1 \%)$.

\section{Instrumentos de evaluación}

Índice Internacional de la Función Eréctil (Rosen et al. 1997), que sirve para medir el nivel de función sexual en hombres. Está formado por 15 ítems que se responden en función de una escala de respuesta de tipo Likert que oscila entre 0 y 5 , dependiendo de la frecuencia en la que se realizan las acciones descritas en el enunciado. El sumatorio de las puntuaciones de los distintos ítems se agrupan en 5 factores: función eréctil, que es la capacidad de erección del hombre durante la relación sexual; función orgásmica, que es la facilidad para alcanzar el orgasmo durante la relación sexual; deseo sexual, que es el impulso a la búsqueda de relaciones sexuales en otros; satisfacción sexual, que es el grado de satisfacción subjetiva con respecto a sus relaciones sexuales, y satisfacción general, que es el grado de satisfacción subjetiva general sobre todos los aspectos de la vida. Cuanto mayor sean las puntuaciones en los distintos factores, mayor será el desempeño en la fase que se evalúa. Con la muestra de este estudio el instrumento muestra una alta fiabilidad $(\alpha=0,952)$, cercana a la del instrumento original $(0,91)$.

Índice de Función Sexual Femenina (Rosen et al. 2000), que sirve para medir el nivel de función sexual en mujeres. Es una escala tipo Likert formada por 19 ítems. Se valora cada ítem del 0 al 5 dependiendo de la frecuencia en la que se realizan las acciones descritas en el enunciado del ítem. El sumatorio de las puntuaciones de los distintos ítems se agrupan en 6 factores: deseo sexual, referido al impulso a la búsqueda de relaciones sexuales en otros; excitación subjetiva, referida a la capacidad de excitación percibida durante las relaciones sexuales; función orgásmica, referida a la facilidad para alcanzar el orgasmo durante la relación sexual; lubricación, referida a la cantidad de segregación de flujo vaginal durante las relaciones sexuales; satisfacción con respecto a las relaciones sexuales realizadas y dolor, que es la cantidad de dolor registrado por el paciente durante las relaciones sexuales. Cuanto mayor sean las puntuaciones en los distintos factores, mayor será la función sexual del sujeto. Con la muestra de este estudio el instrumento muestra una alta fiabilidad $(\alpha=0,964)$, similar a la del instrumento original $(0,97)$.

Además, el nivel de estrés se midió a través de un ítem elaborado ad hoc: "¿Actualmente, qué nivel de estrés diría que sufre en su vida diaria?». Los participantes podían responder a esta pregunta en una escala de 0 a 10 , representando 0 el nivel más bajo de estrés percibido y 10 el más alto.

\section{Procedimiento}

Se trata de un estudio transversal correlacional en el que se pretendía evaluar la influencia del estrés en la población joven adulta, tanto en hombres como en mujeres. En primer lugar, se seleccionaron los instrumentos de evaluación que mejor medían las variables objeto de estudio. La batería de evaluación se diseñó en formato en línea por la comodidad, eficiencia y menor deseabilidad social que tiene esta modalidad de administración, la cual tenía una duración aproximada de 10 minutos. La difusión se realizó durante 2 meses a través de las redes sociales más utilizadas en este grupo poblacional. En el cuestionario se incluía una presentación, en la que aparecía una breve descripción del objetivo del trabajo, el motivo de la realización del mismo, así 
como la duración estimada de cumplimentación, el compromiso de confidencialidad y el consentimiento informado.

\section{Análisis de datos}

Los datos se analizaron mediante el programa SPSS Statistics 25. En el análisis descriptivo se calcularon medias y desviaciones típicas para las variables cuantitativas y frecuencias para variables cualitativas. Por otra parte, se realizó una prueba t para analizar la diferencia en el nivel de estrés entre dos grupos (hombres y mujeres). $Y$, por último, se realizó una correlación de Pearson para analizar la relación de nivel de estrés con cada una de las dimensiones de la función sexual masculina y femenina.

\section{Resultados}

Nivel autopercibido de estrés: diferencias de género

El nivel medio de estrés que los participantes indican que experimentan en la vida diaria es de 5,20 ( $D T=2,50)$ sobre un máximo de 10. Las mujeres identifican puntuaciones medias de estrés ligeramente superiores $(M=5,37 ; D T=2,50)$ a los hombres $(M=5,10 ; \quad D T=2,51)$, no encontrándose diferencias estadísticamente significativas $(t=-1,542 ; p=0,124)$. Atendiendo a la distribución de los niveles de estrés, no se muestran diferencias estadísticamente significativas (chi cuadrado=16,738; $\mathrm{p}=0,080$ ). No obstante, un mayor número de mujeres que de hombres informa de niveles de estrés de 8 o más $(22,3 \%$ frente a $17,7 \%)$ (véase la figura 1$)$.

\section{- Hombre Mujer}

$100 \%$

$75 \%$

$50 \%$

$25 \%$

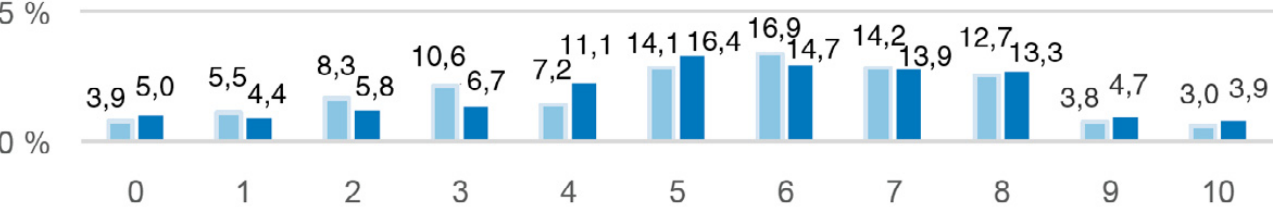

Figura 1. Distribución del nivel de estrés informado en hombres y mujeres.

La influencia del nivel de estrés en la función sexual femenina

El nivel de estrés que las mujeres jóvenes perciben se relaciona de manera estadísticamente significativa con signo negativo con la puntuación total de la función 
sexual femenina $(r=-0,182 ; p=0,001)$. Además, como se muestra en la tabla 1 , también aparecen relaciones significativas a nivel estadístico con todas las dimensiones que componen la respuesta sexual femenina, excepto con el deseo sexual.

Tabla 1

Análisis de correlaciones entre el nivel de estrés percibido y las dimensiones de la función sexual femenina

\begin{tabular}{cccccccc}
\hline & & $\begin{array}{c}\text { Deseo } \\
\text { sexual }\end{array}$ & $\begin{array}{c}\text { Excitación } \\
\text { subjetiva }\end{array}$ & Lubricación & $\begin{array}{c}\text { Función } \\
\text { orgásmica }\end{array}$ & Satisf. & Dolor \\
\hline $\begin{array}{c}\text { Nivel } \\
\text { de }\end{array}$ & $r$ & $-0,071$ & $\mathbf{- 0 , 1 9 2}$ & $\mathbf{- 0 , 1 5 8}$ & $\mathbf{- 0 , 1 6 4}$ & $\mathbf{- 0 , 1 5 4}$ & $\mathbf{- 0 , 1 2 0}$ \\
estrés & $p$ & 0,200 & $\mathbf{5 0 , 0 0 1}$ & $\mathbf{0 , 0 0 4}$ & $\mathbf{0 , 0 0 3}$ & $\mathbf{0 , 0 0 5}$ & $\mathbf{0 , 0 2 9}$ \\
& & & & & & & \\
\hline
\end{tabular}

La influencia del nivel de estrés en la función sexual masculina

En el caso de los hombres jóvenes, el nivel de estrés autopercibido no se relaciona de manera estadísticamente significativa con la puntuación total de la función sexual masculina $(r=-0,072 ; p=0,083)$. Tampoco aparecen relaciones significativas a nivel estadístico con las dimensiones que componen la respuesta sexual masculina (véase la tabla 2), excepto con la satisfacción general.

Tabla 2

Análisis de correlaciones entre el nivel de estrés percibido y las dimensiones de la función sexual masculina

\begin{tabular}{ccccccc}
\hline & & $\begin{array}{c}\text { Función } \\
\text { eréctil }\end{array}$ & $\begin{array}{c}\text { Función } \\
\text { orgásmica }\end{array}$ & Deseo sexual & $\begin{array}{c}\text { Satisfacción } \\
\text { sexual }\end{array}$ & $\begin{array}{c}\text { Satisfacción } \\
\text { general }\end{array}$ \\
\hline $\begin{array}{c}\text { Nivel } \\
\text { de }\end{array}$ & $\mathrm{r}$ & $-0,060$ & $-0,045$ & $-0,008$ & $-0,077$ & $\mathbf{- 0 , 0 9 7}$ \\
estrés & $\mathrm{p}$ & 0,148 & 0,283 & 0,841 & 0,066 & $\mathbf{0 , 0 2 0}$ \\
\hline
\end{tabular}

\section{Discusión y conclusiones}

En los últimos años se ha producido una proliferación de investigaciones que relacionan el estrés con la sexualidad de las personas por el gran impacto sobre la calidad de vida. El presente estudio busca ampliar el conocimiento acerca de esta relación en la salud sexual masculina y femenina. Efectivamente, nuestros hallazgos van en consonancia con aquellas investigaciones cuyos resultados señalan una influencia negativa del estrés diario en la salud sexual, como aparece en la revisión sistemática realizada por Galanakis et al. (2015). Sin embargo, se observa un efecto diferencial del estrés en función del género, siendo la sexualidad de las mujeres la más afectada ante niveles elevados de estrés. Como se demuestra en el estudio de Ip, Lindfelt, Tran, Do y Barnett (2018), las mujeres presentan un mayor nivel de estrés autopercibido. Además, otros estudios indican que estas diferencias podrían explicarse 
por el uso diferencial de estrategias de afrontamiento del estrés. Por ejemplo, González Cabanach, Fariña, Freire, González y Ferradás (2013) realizaron una investigación con una muestra de más de 2000 estudiantes y demostraron que las mujeres recurrían principalmente a estrategias de afrontamiento de tipo emocional, como la búsqueda de apoyo, y los hombres de tipo cognitivo, como la reevaluación cognitiva. Las mujeres, por tanto, utilizan en mayor medida estrategias centradas en el manejo emocional, mientras que los hombres estrategias enfocadas en modificar el significado de la situación estresante. También desde el punto de vista neurológico podría explicarse esta afectación diferencial del estrés en la sexualidad. En el estudio realizado por Wang et al. (2007) se observó cómo en los hombres la presencia de estrés producía una asimetría en cuanto a la actividad del córtex prefrontal, mientras que las áreas que se activaron en primer lugar en las mujeres fueron las áreas que conforman el sistema límbico. Además, la asimetría producida en el córtex prefrontal de los hombres correlacionaba con el aumento de los niveles de cortisol, mientras que la activación del sistema límbico de las mujeres no tenía una relación tan consistente con los niveles de esta hormona. Los autores del estudio, basándose en la teoría fight or flight y tend and befriend (Taylor et al. 2000), sugieren que estas diferencias a nivel neural pueden deberse a una herencia filogenética.

En cuanto a la sexualidad femenina en concreto, el estrés repercute negativamente en todos los componentes de la función sexual a excepción del deseo sexual. Quizá esto pueda ser explicado por el mayor componente psicológico que tiene la fase del deseo sexual en comparación con el mayor componente fisiológico del resto de fases de la respuesta sexual femenina. Estos resultados van en consonancia con los resultados obtenidos en el estudio de Hamilton y Meston (2013), los cuales, monitorizaron los niveles de activación genital y psicológica ante el visionado de material erótico y observaron que las mujeres con un nivel de estrés elevado mostraron menor activación genital pero no psicológica. Los autores explicaron estos resultados en base a la acción del cortisol.

Por último, en relación con la función sexual masculina, resulta curioso que los resultados de nuestro estudio indiquen que el estrés únicamente repercute de manera negativa en una de sus dimensiones, concretamente en la satisfacción general. Estos resultados son contrarios a los obtenidos en el estudio de Bodenmann, Ledermann, Blattner y Galluzzo (2006), donde se aprecia una repercusión negativa en otros componentes de la función sexual, como el deseo sexual, dando lugar a problemas tales como el deseo sexual hipoactivo. Estas diferencias entre estudios pueden deberse a distintos factores. Por una parte, en el estudio mencionado, se relaciona el estrés con determinadas disfunciones sexuales, mientras que, en el presente estudio, se han tenido en cuenta los distintos componentes que conforman la función sexual. Por otra parte, la media de edad en ambos estudios es completamente distinta: en el presente estudio han participado jóvenes y en el estudio anteriormente citado participaron mayoritariamente adultos mayores. En relación con la satisfacción sexual general, cabe destacar que son escasos los estudios que analizan esta variable, ya que gran parte de la literatura científica ha centrado sus esfuerzos en el estudio de variables mayormente fisiológicas de la respuesta sexual y no tanto en indagar en las variables cognitivas y en la experiencia subjetiva de la propia actividad sexual (PérezTriviño 2013).

Para finalizar, este estudio permite considerar la relevancia del estrés en la función sexual de los jóvenes adultos, principalmente en las mujeres. No obstante, existen limitaciones que necesitan ser mejoradas en futuras investigaciones. Por ejemplo, haber utilizado como medida de evaluación del nivel de estrés un único ítem y no un instrumento de evaluación validado. Pese a ello, cabe destacar la importancia que tiene la autopercepción del nivel de estrés percibido por parte de la propia persona. 
Por otra parte, dado que se han utilizado medidas de evaluación diferentes para hombres y mujeres, no se han podido analizar las diferencias de género. Por último, sería necesario incluir otras variables que pueden estar modulando la influencia del estrés en la función sexual, como la edad o los estilos de afrontamiento. A pesar de lo anterior, los resultados obtenidos permiten mejorar las estrategias de prevención primaria y secundaria de problemas sexuales. En primer lugar, se considera relevante dotar a los jóvenes de estrategias adaptativas de afrontamiento del estrés que minimicen la interferencia en la calidad de vida sexual y, en segundo lugar, resulta fundamental la detección precoz de población en riesgo, así como incluir los problemas de estrés en las evaluaciones de las dificultades sexuales.

\section{Referencias bibliográficas}

Abedi, Parvin, Mahnaz Afrazeh, Nahid Javadifar y Azadeh Saki. 2015. "The relation between stress and sexual function and satisfaction in reproductive-age women in Iran: a cross-sectional study». Journal of sex \& marital therapy, 41(4): 384390.

Bodenmann, Guy, David Atkins, Marcel Schär y Valérie Poffet. 2010. "The association between daily stress and sexual activity». Journal of Family Psychology, 24(3): 271-279.

Bodenmann, Guy, Thomas Ledermann, Denise Blattner y Claudia Galluzzo. 2006. «Associations among everyday stress, critical life events, and sexual problems». The Journal of nervous and mental disease, 194(7): 494-501.

Crisp, Catrina, Christine Vaccaro, Apurva Pancholy, Steve Kleeman, Angela Fellner y Rachel Pauls. 2013. "ls female sexual dysfunction related to personality and coping? An exploratory study». Sexual medicine, 1(2): 69-75.

Duque Moreno, Aranzazu. 2017. Efectos del estrés social sobre la memoria y su modulación farmacológica en ratones. Tesis doctoral. Valencia: Universidad de Valencia.

Galanakis, Michael, Maria Despoina Kallianta, Chrysoula Katsira, Dimitra Liakopoulou, George Chrousos y Christina Darviri. 2015. "The association between stress and sexual dysfunctionality in men and women: A systematic review». Psychology, 6(14): 1888-1892.

García-Herrero, Susana, Miguel Ángel Mariscal, José Manuel Gutiérrez y Dale Ritzel. 2013. "Using Bayesian networks to analyze occupational stress caused by work demands: Preventing stress through social support». Accident Analysis \& Prevention, 57: 114-123.

Gianaros, Peter y Tor Wager. 2015. «Brain-body pathways linking psychological stress and physical health». Current directions in psychological science, 24(4): 313321.

González Cabanach, Ramón, Francisca Fariña, Carlos Freire, Patricia González y María del Mar Ferradás. 2013. "Diferencias en el afrontamiento del estrés en estudiantes universitarios hombres y mujeres». European Journal of Education and Psychology, 6(1): 19-32.

Hamilton, Lisa Dawn y Cindy Meston. 2013. "Chronic stress and sexual function in women». The journal of sexual medicine, 10(10): 2443-2454.

Hostinar, Camelia, Robin Nusslock y Gregory Miller. 2018. «Future directions in the study of early-life stress and physical and emotional health: implications of the neuroimmune network hypothesis». Journal of Clinical Child \& Adolescent Psychology, 47(1): 142-156.

Ip, Eric, Tristan Lindfelt, Annie Tran, Amanda Do y Mitchell Barnett. 2018. «Differences in Career Satisfaction, Work-life Balance, and Stress by Gender in a National Survey of Pharmacy Faculty». Journal of pharmacy practice, 1-5. 
Kelley, Erika y Christine Gidycz. 2019. «Posttraumatic stress and sexual functioning difficulties in college women with a history of sexual assault victimization». Psychology of Violence, 9(1): 98-107.

Kirby, Elizabeth Diana. 2011. Stress effects on adult hippocampal neurogenesis and sexual reproductive function. Tesis doctoral. Berkeley, California: Universidad de California.

Leavitt, Chelom, Brandon McDaniel, Megan Maas y Mark Feinberg. 2017. «Parenting stress and sexual satisfaction among first-time parents: A dyadic approach». Sex roles, 76(5-6): 346-355.

Pérez Triviño, Fátima. 2013. Nueva escala de satisfacción sexual (NSSS) en usuarios de redes sociales. Tesis doctoral. Almería: Universidad de Almería.

Romero Martín, Manuel. 2009. Implicaciones de la respuesta de estrés sobre el proceso de estudio en estudiantes de Ciencias de la Salud. Tesis doctoral. A Coruña: Universidad de A Coruña.

Rosen, Raymond, Candice Brown, Julia Heiman, Sandra Leiblum, Cindy Meston, Ridwan Shabsigh, Dean Ferguson y Riccardo D'Agostino. 2000. "The Female Sexual Function Index (FSFI): a multidimensional self-report instrument for the assessment of female sexual function». Journal of sex \& marital therapy, 26(2): 191-208.

Rosen, Raymond, Alan Riley, Gorm Wagner, lan Osterloh, John Kirkpatrick y Avanish Mishra. 1997. "The international index of erectile function (IIEF): a multidimensional scale for assessment of erectile dysfunction». Urology, 49(6): 822-830.

Selye, Hans. 1936. "A syndrome produced by diverse nocuous agents». Nature, 138(3479): 32.

Shifren, Jan, Brigitta Monz, Patricia Russo, Anthony Segreti y Catherine Johannes. 2008. "Sexual problems and distress in United States women: prevalence and correlates». Obstetrics \& Gynecology, 112(5): 970-978.

Taylor, Shelley, Laura Cousino Klein, Brian Lewis, Tara Gruenewald, Regan Gurung y John Updegraff. 2000. «Biobehavioral responses to stress in females: tend-andbefriend, not fight-or-flight». Psychological review, 107(3): 411-429.

Thoits, Peggy. 2010. "Stress and health: Major findings and policy implications». Journal of health and social behavior, 51(supl.): 41-53.

Wang, Jiongjiong, Marc Korczykowski, Hengyi Rao, Yong Fan, John Pluta, Ruben Gur, Bruce McEwen y John Detre. 2007. "Gender difference in neural response to psychological stress». Social cognitive and affective neuroscience, 2(3): 227239. 